\title{
Cleaning potential of different air abrasive powders and their impact on implant surface roughness
}

\author{
Victor H. Matsubara DDS, MSc, $\mathrm{PhD}^{1}$ @ | Bron W. Leong DMD ${ }^{1} \quad$ | \\ Marcus J. L. Leong DMD ${ }^{1}$ @ | Zacharij Lawrence $\mathrm{DMD}^{1}$ ｜ Thomas Becker PhD ${ }^{2}$ @ | \\ Alessandro Quaranta DDS, $\mathrm{PhD}^{3}$ (1)
}

\footnotetext{
${ }^{1}$ Faculty of Health and Medical Sciences, Dental School, University of Western Australia, Perth, Western Australia, Australia

${ }^{2}$ Faculty of Science and Engineering, School of Molecular and Life Sciences, Curtin University, Perth, Western Australia, Australia

${ }^{3}$ School of Dentistry and Oral Health, Griffith University, Gold Coast, Queensland, Australia

Correspondence

Victor H. Matsubara, Faculty of Health and Medical Sciences, Dental School, University of Western Australia, 17 Monash Avenue, Nedlands, Perth, WA 6009, Australia. Email: victor.matsubara@uwa.edu.au
}

\begin{abstract}
Background: Implant surface roughness after air abrasive therapy has not been measured precisely in previous research. Debridement with air abrasion facilitates the mechanical removal of bacterial biofilms but may damage implant surfaces on a microscopic level.

Purpose: This study aimed to investigate the cleaning potential of various air abrasive powders and their effect on titanium implant surfaces.

Materials and Methods: Twenty implants coated with red ink were inserted into three-dimensional printed circumferential bone defect models. Treatment was completed with three types of air abrasive powders: sodium bicarbonate (SB), glycine, and erythritol for 60 seconds. Water alone was used as control. The percentage of remaining ink was assessed using digital photography and graphic software. Implant surface topography/roughness was quantified using optical profilometry and examined via scanning electron microscopy. The microscopic analysis was performed at two implant areas: collar (Laser-Lok surface) and threads.

Results: The cleaned surfaces (\%, mean \pm SD) after treatment with SB, glycine, and erythritol accounted for $49.3 \pm 3.6 \%, 33.1 \pm 1.2 \%$, and $25.1 \pm 0.7 \%$, respectively. Statistically significant differences were found between all groups $(P<.001)$. SB was the only powder that significantly increased the implant roughness $\left(S_{a}\right)$ on both the implant collar (1.53-2.10 $\mu \mathrm{m})$ and threads (3.53-4.20 $\mu \mathrm{m})$. Regardless of the abrasive powder used, the collar, emerging implant surfaces from the defect base, and surfaces beneath implants threads exhibited more post-treatment residual ink.

Conclusion: Large-sized powder showed the greatest cleaning capacity, but caused more alterations to the implant surface. Glycine and erythritol displayed no significant changes in surface roughness, however, demonstrated a limited ink removal capacity.
\end{abstract}

\section{KEYWORDS}

air abrasion, decontamination, dental implants, peri-implantitis 


\section{1 | INTRODUCTION}

Dental implants are often utilized in modern dentistry as a reliable treatment option for supporting and retaining various types of dental prosthesis; from single units to full-arch reconstruction. Success rates of $95 \%$ have been reported in healthy patients. ${ }^{1}$ However, the selection of suitable patients for implant treatment is often overlooked as implants are being inserted in patients with poor oral hygiene or periodontal disease without receiving proper treatment or guidance on preventative oral hygiene instruction.

Prospective studies have noted that failures of osseointegrated implants occurring after successful prosthetic reconstruction are mainly due to biological complications (peri-implant infections) rather than traumatic forces from occlusal overload. ${ }^{2,3}$ As successfully integrated implants maintain their clinical stability even in advanced cases of peri-implantitis, the most reliable means for diagnosing secondary failure of osseointegration are based on bleeding on probing, probing depths, and assessment using oral radiographs. ${ }^{4}$

The rate of progression of peri-implant diseases associated with plaque accumulation seems to be more pronounced and extensive in dental implants than in natural teeth. ${ }^{5}$ Once bacterial biofilms accumulate on the surface of the implant-supported prosthesis, they promote the disruption of the peri-implant keratinized mucosa, ${ }^{6}$ which protects the bony tissues surrounding the implant body. ${ }^{7}$ This process facilitates the contamination of rough implant surfaces by periodontal pathogens that perpetuate or aggravate peri-implant diseases and intrabony defects, ${ }^{8}$ which are usually circumferential, taking the shape of a crater. ${ }^{9}$

Hence, early detection and treatment of peri-implant diseases are crucial to the longevity of implants. The recovering of soft tissue health around osseointegrated dental implants can be achieved by daily selfadministered maintenance procedures (mechanical and chemical plaque control) or professional interventions involving mechanical debridement with or without the use of adjunctive antimicrobial agents. ${ }^{10}$ Despite different protocols have been reported, there is no reliable clinical evidence as to which regimens are most effective for long-term maintenance and treatment of peri-implant mucositis. ${ }^{11}$

Once the disease progresses to bone loss, the control of bacterial infection around implants becomes more challenging. Many protocols have been suggested for the treatment of peri-implantitis, including non-surgical and surgical interventions. ${ }^{10}$ In both approaches, the decontamination of the implant surfaces is a prerequisite for treatment success, and so far the implant cleaning involved mechanical debridement using curettes and ultrasonic devices, ${ }^{12}$ or alternative approaches such as air abrasive therapies ${ }^{13}$ and laser application. ${ }^{14}$ Local antimicrobial agents have also been applied as an adjunct to manual debridement and have demonstrated significant improvement for changes in probing attachment levels and probing pocket depth. ${ }^{15}$

Curettes and ultrasonic devices are commonly used to clean contaminated implants; however, they have potential to damage the titanium surface, ${ }^{16}$ and seem to fail to restore the biocompatibility of rough titanium surfaces. ${ }^{17}$ In turn, different types of laser have been used in peri-implant therapy: diode, carbon dioxide $\left(\mathrm{CO}_{2}\right)$, neodymium-doped yttrium aluminum garnet, and erbium- doped yttrium aluminum garnet lasers. Despite the variety of equipment available, published meta-analyses have not reported statistically significant differences in pocket reduction and clinical attachment gain between lasers and mechanical debridement alone. ${ }^{14}$ In this scenario, air abrasive protocols are gaining popularity in the treatment of peri-implant diseases.

Although there is no robust clinical evidence suggesting which modality is the most effective for treating peri-implantitis, ${ }^{12}$ in vitro tests have demonstrated that air abrasive powders have superior or equal potential of decontamination when compared to other cleaning modalities. ${ }^{18,19}$ The use of small-sized air abrasive powders was found to be less damaging to implant surfaces than other cleaning methods, ${ }^{19}$ and may also combine both the advantages of mechanical and chemical debridement by incorporating chlorhexidine into their powder formulation. This combination has demonstrated a cleaning efficiency of up to $85 \%$, suggesting that air abrasion is an effective technique for implant surface decontamination for supragingival sites with shallow 4 to $5 \mathrm{~mm}$ pockets as well as cases of open flap debridement. $^{13}$

The surgical approach, in turn, is indicated when there is advanced bone loss around implants. The open flap debridement was found to be more effective than nonsurgical therapies in the short term, promoting a significantly higher reduction in peri-implant probing depth. ${ }^{20}$ This is likely due to increased access and visibility of implant surfaces. Different adjunctive treatments to open flap periimplant debridement are available, including systemic antibiotics ${ }^{21}$ and bone augmentation procedures. ${ }^{12}$ The latter requires an effectively cleaned surface with no change in the implant surface micromorphology in order not to interfere with the biocompatibility. in vitro tests have shown that air abrasion therapy using small-sized powders provides better cleaning capacity compared to both curettes and ultrasonic tips in open flap debridement simulation, using periimplantitis bony defect models. ${ }^{18}$

Despite the surgical approach associated with air abrasive therapy currently being one of the best options available for implant surface decontamination, there is currently no consensus on the most effective air abrasive powder for treatment. Previous research on air abrasive therapies for implant decontamination has largely been limited to in vitro studies focusing on the cleaning of titanium disks, which do not contain the screw component found in dental implants and failed to consider bone defect anatomy in late stage periimplantitis. ${ }^{22} \mathrm{~A}$ handful of studies tried to simulate open flap air abrasive therapy using real implants in a simulated model of periimplantitis. ${ }^{18,23,24}$ However, none had utilized quantitative analysis to assess both cleaning efficiency and implant roughness when comparing different air abrasive powders. Different areas of the implant, such as the collar and threads, were not individually assessed in these studies. Hence, this study aimed to address these gaps in knowledge by comparing the cleaning capacity of small (glycine and erythritol) and larger sized (sodium bicarbonate, SB) air abrasive particles, as well as quantifying their impact on implant roughness and morphology in different areas of the implant using a standardized bone defect model. 


\section{2 | MATERIALS AND METHODS}

\section{1 | Experimental design}

This in vitro study consisted of four experimental groups: glycine, erythritol, sodium bicarbonate, and water (control group). Air abrasive therapies were conducted with the implants inserted in a threedimensional (3D) printed bone defect, leaving the collar and the first three threads exposed within the defect. The cleaning capacity of each group was quantitatively analyzed using a validated ink model of implant biofilm, whereas the implant roughness changes were qualitative and quantitatively assessed via scanning electron microscopy (SEM) and optical profilometry, respectively. After each treatment, the implant samples were removed from the defects and assessed for changes in roughness, followed by the ink removal quantification. Direct contact with the implant surface was avoided throughout the study phases.

\section{2 | Implants and bone defect model}

Twenty tapered bone-level BioHorizons titanium implants $(4.6 \mathrm{~mm}$ diameter, $12 \mathrm{~mm}$ height) were used in the study, with five allocated to each group. This implant was chosen for its Laser-Lok (BioHorizon; $1.5 \mathrm{~mm}$ high) precision-engineered structure designed specifically on the implant collar to produce a nanostructure surface, different to that of the thread surface (Figure 1).

The 3D printed defects were fabricated to simulate circumferential bone resorption morphology that mimicked advanced stages of peri-implantitis. The defect was fabricated to a dimension of $6 \mathrm{~mm}$ in depth and $9 \mathrm{~mm}$ in width with consideration to the size of the implant, simulating a class le defect ${ }^{9}$ that is relatively common around implants and challenging to clean. The circumferential shaped defect represents

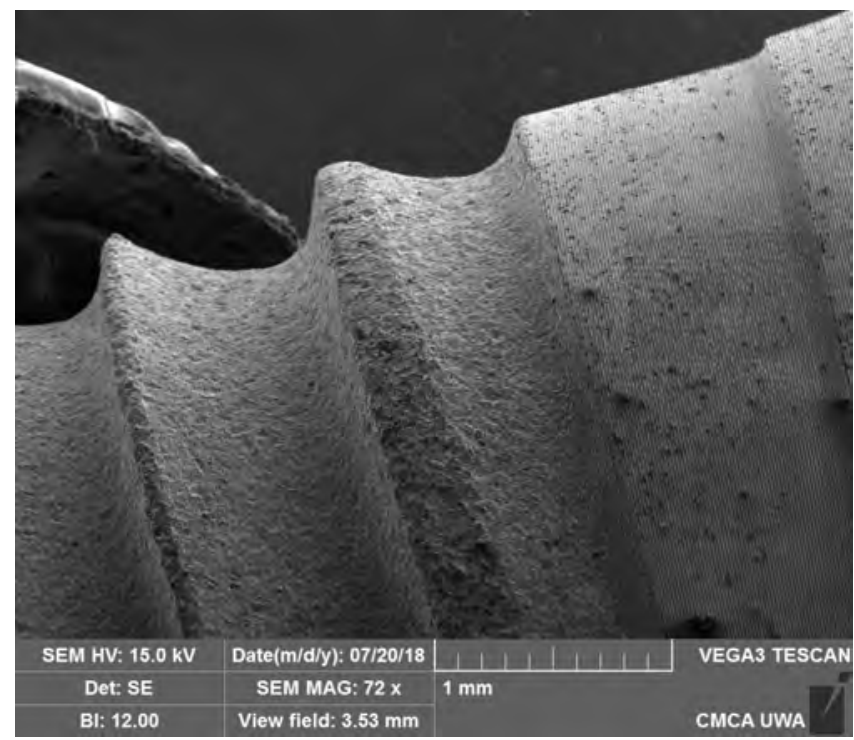

FIGURE 1 Scanning electron microscopy image of BioHorizon implant highlighting the two distinguishable types of implant surfaces the physical environment of peri-implantitis and this was executed via digital scanning (R1000 3D Scanner; 3Shape, Australia) of a circumferential defect template created in die stone followed by 3D printing (Cara Print 4.0 3D Printer; Kulzer, Australia) in resin (dima Print Cast; Kulzer) at $30 \mu \mathrm{m}$ increments. This material has a high melting point and a high water and chemical resistance, making it the ideal material for implant site preparation using drills. The application of standardized 3D printed models ensured a new level of accuracy and uniformity between trials.

The implant site preparation was performed according to the manufacturer's instructions using BioHorizon dental drills along with the Nobel Biocare OsseoSet 200 Dental Implant System at 450 rpm. The final implant position was established with the exposure of the first three coronal threads whilst ensuring the entire implant was contained within the circumferential defect (Figure 2).

\subsection{Air abrasive treatment}

All air abrasive treatments were conducted by the same experienced clinician using AIRFLOW Master system (EMS, Nyon, Switzerland) with a supragingival tip. The test groups were treated using SB (40-65 $\mu \mathrm{m}$ Classic Comfort; EMS), glycine powder (25 $\mu \mathrm{m}$ Perio Powder; EMS), and erythritol powder (14 $\mu \mathrm{m}$ Air-flow Plus Powder; EMS). The control group received water spray treatment without powder. The water pressure was set at 4.5 to $6.5 \mathrm{psi}$ and air pressure at 5.5 to $7.5 \mathrm{psi}$, and the treatment time was limited to 60 seconds. Factors that were not standardised included the angulation of the application piece and working distance as they varied according to the area being cleaned.

\subsection{Ink removal assessment}

Prior to placing into the defect, all implants were uniformly coated with one layer of red, non-water soluble ink (Sharpie Fine Point Permanent Marker) to represent an optically identifiable plaque accumulation (Figure 3A). The implant was removed from the bone defect after each treatment and dried with compressed air for 10 seconds to remove any remaining powder (Figure $3 \mathrm{~B}$ ).

A total of 12 standardized digital photographs of the implant were taken using a DSLR camera (Canon DSLR fitted with a macro lens) as it revolved around a base with 12 evenly marked intervals
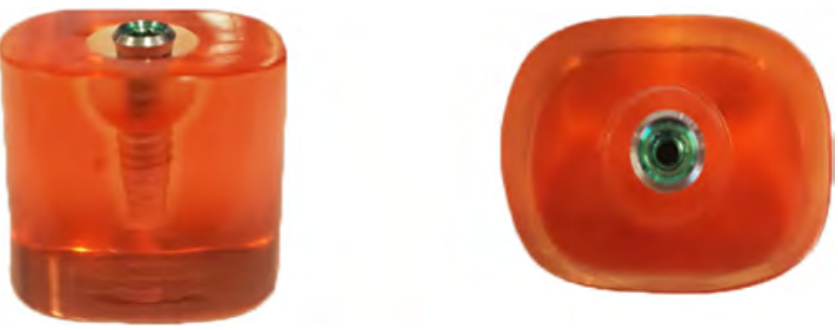

FIGURE 2 Three-dimensional printed circumferential bone defect model with implant inserted after implant site preparation 
(A)

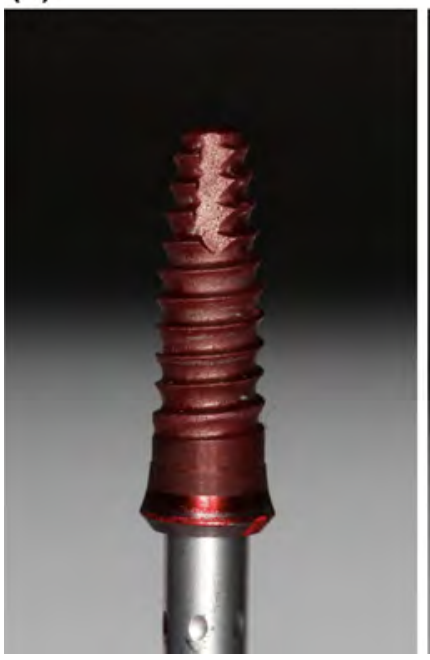

(B)

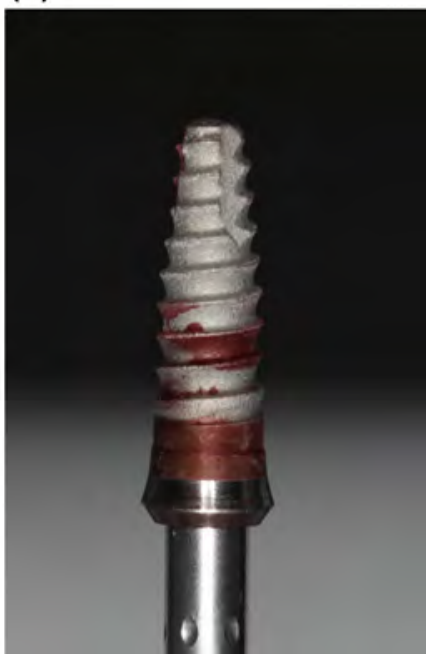

FIGURE 3 A, Implant coated with red ink before treatment. B, Implant coated with residual red ink after treatment

(A)

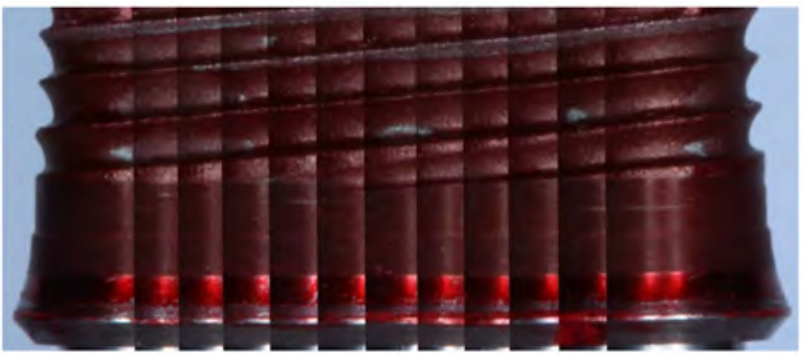

(C)

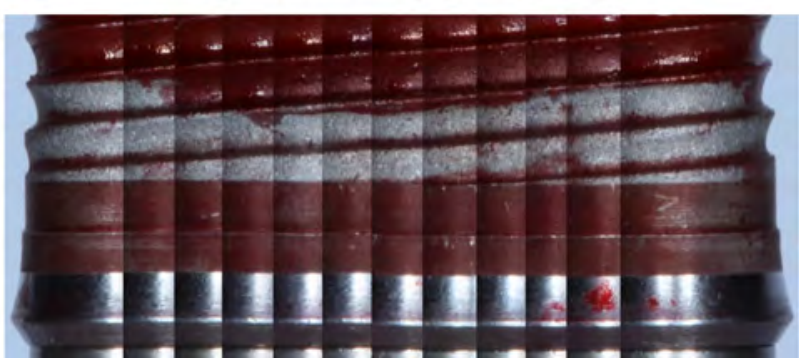

(B)

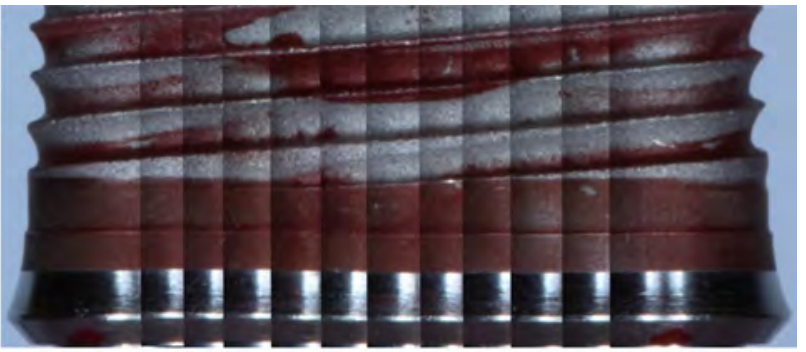

(D)

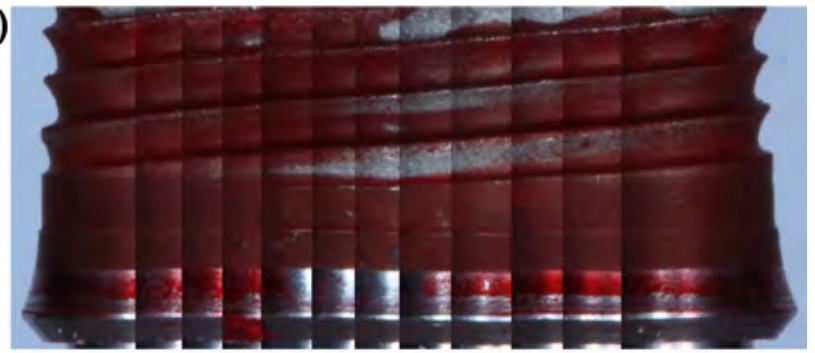

FIGURE 4 Collated digital photographs (panoramic) of implant samples showing red ink removal after 60 seconds of air abrasive treatment. A, Control-water with no powder. B, Sodium bicarbonate. C, Glycine. D, Erythritol

( $30^{\circ}$ intervals). Aperture was set at F32 with the shutter speed at 1/4000. The photographs were compounded to form a panoramic image of the implant (Figure 4). The pixel percentage of the remaining ink was calculated with the help of a visual color analysis software (Photoshop CC Software; Adobe Systems Software, California) (Figure 5). These findings were then compared to the initial data obtained prior to treatment and the percentage of ink removal up to the third thread was then calculated.

\section{5 | Implant surface analysis}

The surface topography was measured using a new optical profilometry analysis via TrueSurface Microscopy that utilizes white light interferometry (WITec Alpha 300; WITec, Ulm, Germany). The results produced were of area roughness $\left(S_{a}\right)$. The values for $S_{a}$ were obtained through evaluation of changes in heights on the implant surface across various individual points. These heights were plotted on profilometry graphs (Figure 6), which allowed for individual analysis of the collar and threads of individual implant samples, before and after air abrasive therapy. Three fields in each the collar and the threads were measured in each sample, and their average was used for statistical analysis. This method allowed more precise surface analysis through acquisition of real 3D data and comparison via graph superimposition. These data were used in conjunction with images obtained via SEM analysis, which provided a visual and qualitative representation of the visible changes on the implant surfaces before and after treatment. 
(A)

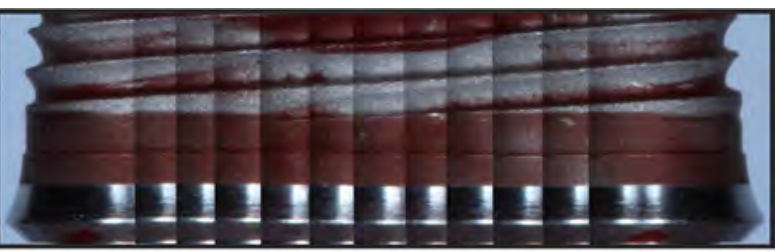

(B)

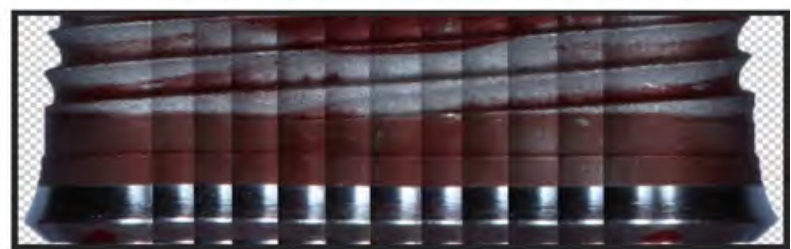

(C)

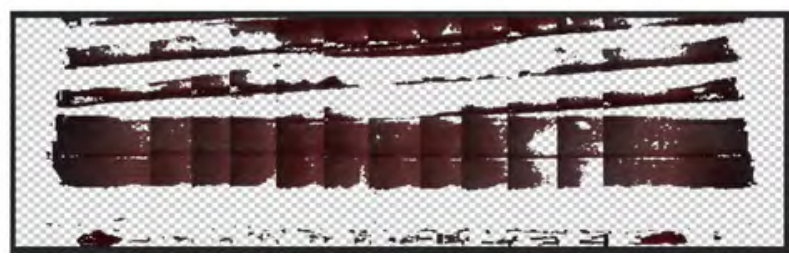

FIGURE 5 Panoramic images of a sample being processed in an image software for the ink removal quantification. A, Original image. B, Removal of the image background. C, Isolation of pixel with the remaining red ink

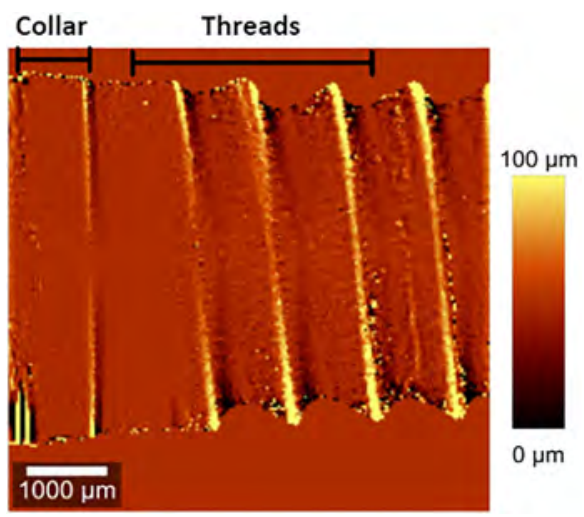

FIGURE 6 Profilometry graphs from TrueSurface Microscopy showing different heights on the implant surface across many individual points. Implant roughness was measured in the implant collar and threads areas

Implant areas such as the collar, thread, and their transitional zones were visualized under a Sigma VP Field Emission Scanning Electron Microscope (Carl Zeiss Inc., Oberkochen, Germany) in high-vacuum mode at $20 \mathrm{kV}, \times 1000$ magnification, and at a $15-\mathrm{mm}$ working distance.

\subsection{Statistical analysis}

A total of five samples for each group of study were performed. Data were expressed as mean \pm SD from three independent experiments. Oneway analysis of variance test followed by Tukey's multiple comparison test were used for statistical analyses. Statistical significance was set at $P<.05$ (GraphPad Prism Version 6.0c; GraphPad Software, La Jolla, California).

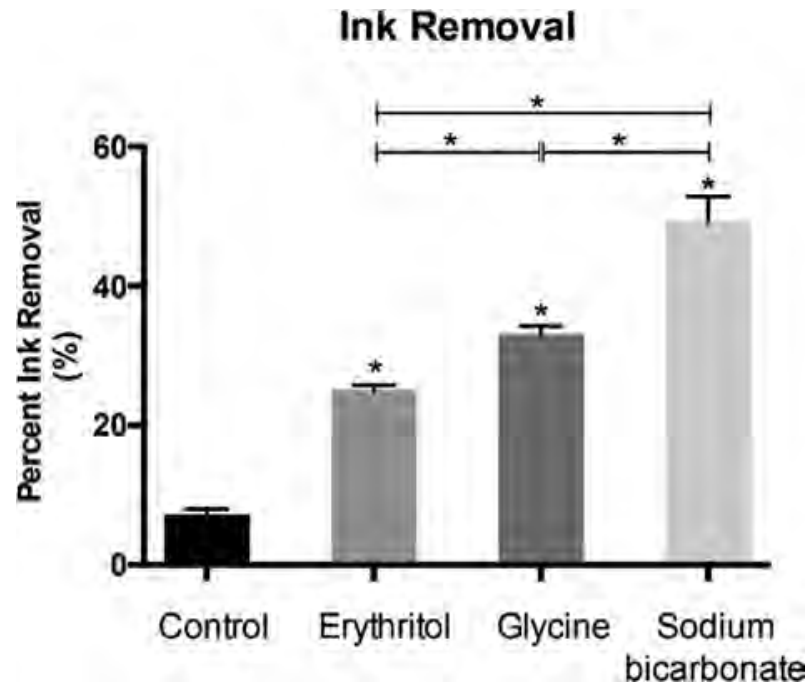

FIGURE 7 Effectiveness of different air abrasive powders in ink removal for implant surface roughness (collar and threads) after 60 seconds of treatment. Data are presented as mean \pm SD $(\mathrm{n}=5) . * P<.001$

\section{3 | RESULTS}

\subsection{Cleaning capacity}

Results obtained from digital photography and subsequent visual color analysis showed statistically significant dye removal between all groups and the control, with SB being the most effective at ink removal, followed by glycine and erythritol, respectively (Figure 7). SB was able to remove $49.3 \pm 3.6 \%$ (mean $\pm S D$ ) of red ink from the collar and first three threads, while the smallest particle, erythritol, had the lowest cleaning capacity removing only $25.1 \pm 0.7 \%$. Glycine cleaned 33.1 $\pm 1.2 \%$. Differences were not only significant between the control and test groups but also among test groups $(P<.001)$.

Panoramic images of the implant samples showed that the most difficult areas to clean using air abrasive treatments were the implant surface near the bottom of the 6-mm defect, the collar (Laser-Lok surface) and beneath implants threads (Figure 4), regardless of the type of abrasive powder used.

\section{2 | Implant surface analysis}

The area roughness parameter $\left(S_{\mathrm{a}}\right)$ measured in the collar and threads of pristine implants were on average 2 and $3.5 \mu \mathrm{m}$, respectively. The treatments with water (control), erythritol, and glycine did not significantly alter the surface roughness in the collar and first three threads, although a slight increase in the $S_{a}$ value (not statistically significant) was observed in the small-sized particle groups (Figure 8). The $S_{a}$ value $(\mu \mathrm{m}$, mean $\pm \mathrm{SD}$ ) in the glycine group varied from $1.59 \pm 0.05$ to $1.70 \pm 0.08 \mu \mathrm{m}$ in the collar, and from $3.34 \pm 0.05$ to $3.40 \pm 0.12 \mu \mathrm{m}$ in the threads. For erythritol, these numbers increased from $1.76 \pm 0.11$ to $1.96 \pm 0.07 \mu \mathrm{m}$ in the collar, and reduced slightly from $3.67 \pm 0.09$ to $3.62 \pm 0.20 \mu \mathrm{m}$ in the threads. 
Implant Collar Roughness

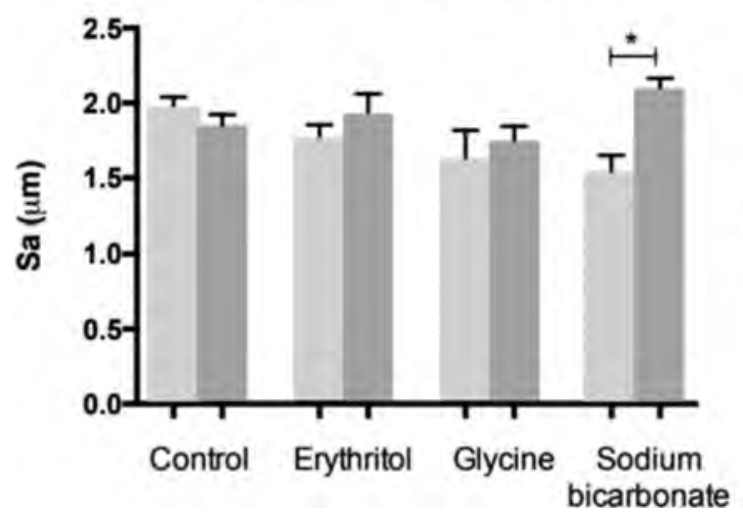

Implant Thread Roughness

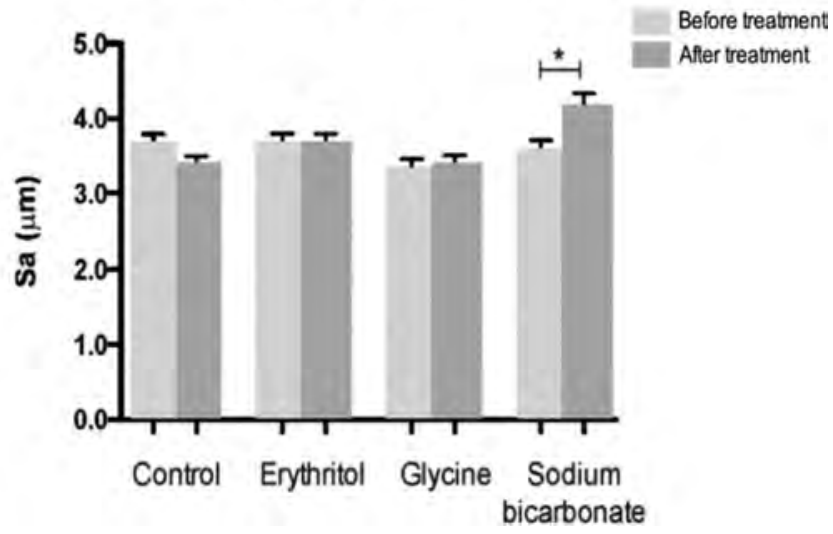

FIGURE 8 Effect of air abrasive powders on titanium implant surface roughness (collar and threads) after 60 seconds of treatment. Data are presented as mean \pm SD $(n=5) . * P<.001$

BEFORE TREATMENT

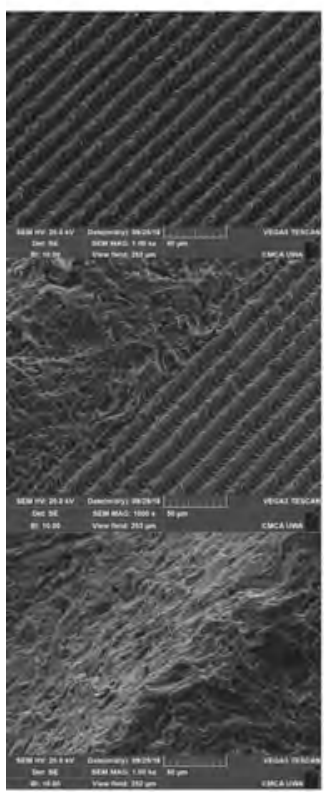

(A)

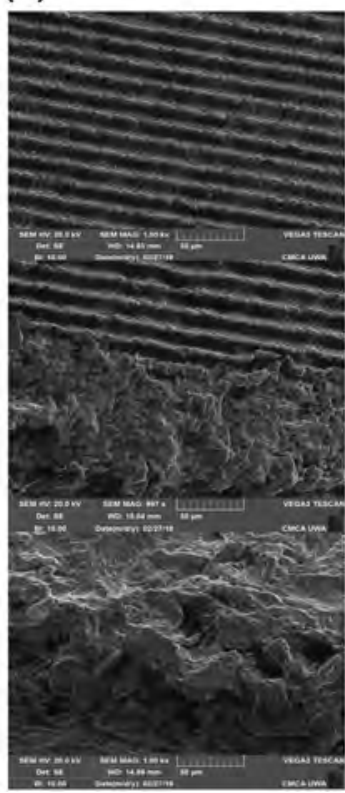

AFTER TREATMENT

(B)

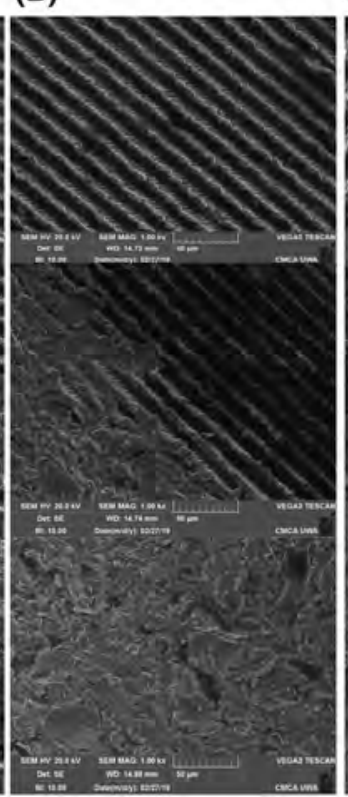

(C)

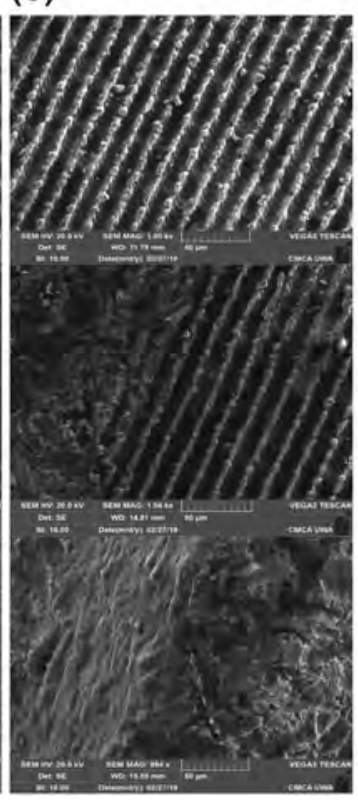

(D)

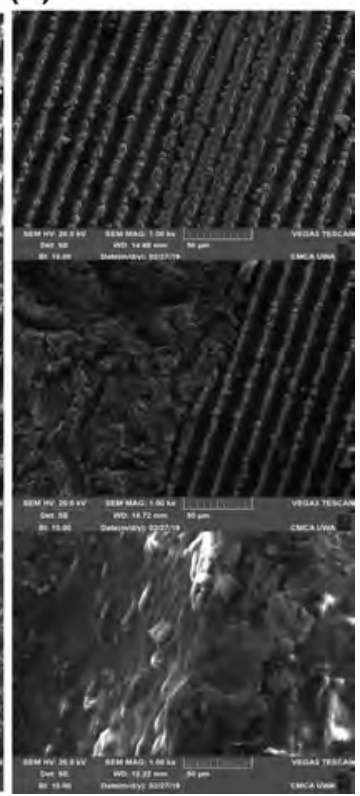

FIGURE 9 Scanning electron microscopy images ( $\times 1000$ magnification) of implants in the collar (Laser-Lok), threads, and the transitional area before and after treatment with. A, sodium bicarbonate. B, glycine. C, erythritol. D, control-water

SB was the only powder that caused significant changes in the topography of implants, increasing the $S_{a}$ value from $1.54 \pm 0.03 \mu \mathrm{m}$ before treatment to $2.10 \pm 0.04 \mu \mathrm{m}$ after treatment in the collar, and from $3.53 \pm 0.20$ to $4.20 \pm 0.15 \mu \mathrm{m}$ in the threads area.

The SEM evaluation provided a clear visual of the implant surfaces, including the unique mechanically manufactured Laser-Lok treatment from BioHorizons (Figure 9). The SEM analysis enhanced the demarcation between the boundaries of the Laser-Lok collar surface and rough thread area. The images obtained before and after treatment were compared and a noticeable obliteration of the transition from the collar to the thread was illustrated on implants treated with SB and glycine. Implants treated with erythritol and water on the other hand showed minimal changes. Subsequently, implants treated with the air abrasive powders resulted in a rougher thread appearance, displaying divots that reduced in magnitude with the reduction in particle size. Images obtained of the control sample lacked definition compared to the samples treated with air abrasion as a result of interference and luminescence of the remaining ink on implant surfaces of the control group.

\section{4 | DISCUSSION}

This in vitro study was the first to combine precise quantitative analyses of the cleaning potential of various air abrasive powders as well 
as their impact on implant surface roughness utilizing a simulation of surgical treatment for peri-implantitis. The decontamination of implant surfaces is fundamental to successful treatment outcomes. This is especially important in a surgical approach that aims to arrest the progression of the pathology by either filling the bone defect created by the peri-implant infection or eliminating the peri-implant pocket through resective procedures. ${ }^{25}$ Current literature demonstrates that air abrasive powders can achieve a level of decontamination comparable to successful guided tissue regeneration procedures. ${ }^{26}$ In addition, powders frequently cause less damage to the implant surface in comparison to hand instruments such as curettes. ${ }^{27}$ However, there is still no consensus on which is the best powder to use.

Larger particles were found to have a good biofilm removal capacity, ${ }^{24}$ although they also cause significant changes on the implant surface. ${ }^{23}$ Smaller particles in turn often create less morphological changes in the implant surface whilst still effectively removing the biofilm. ${ }^{26}$ Despite being frequently used in the management of peri-implant diseases, there is limited evidence in the literature comparing the decontamination of actual implant surfaces with different small-sized air abrasive powders, such as glycine and erythritol, and the morphological change on the implant surface caused by these treatments.

According to our ink removal assessment, smaller abrasive particles resulted in poorer cleaning capacity. This finding is in accordance with a previous in vitro study that also tested the cleaning capacity of air abrasive powders in an open flap decontamination model with various air pressure regulations. ${ }^{23}$ Despite being commonly adopted to assess the cleaning potential of different implant debridement methods in in vitro studies, ${ }^{18,23}$ the ink model used in this study presents limitations. Firstly, the ink may be more difficult to remove than oral biofilms, as in vitro tests using viable bacterial biofilms colonizing the implant surface found equal decontamination capacity of glycine and SB. ${ }^{24}$ This fact, however, does not invalidate our findings because the present study aimed to compare the capacity of different powders with various sizes and shapes to remove external contaminants from the implant surface, not their potential to create a germ-free surface. Another limitation is that the red ink is easier to detect during air abrasive treatment when compared to a clinical situation involving bacterial contamination of implants. This limitation was mitigated by timing each treatment and conducting the experiment on all groups under the same conditions.

To standardize the treatment conditions, a bone defect simulating circumferential bone loss in peri-implantitis was designed and identical copies were 3D printed for implant site preparation. This protocol maintained consistency in implant positioning within the defect and allowed for a more uniform comparison between groups. Through this, it was found that the most difficult areas for cleaning are the apically facing thread surfaces and the area near the base of the defect. A previous study also found residual staining below the threads regardless of treatment approach. ${ }^{28}$ The use of special nozzles for subgingival areas in air abrasive therapy may be the most appropriate alternative to overcome the challenge of decontaminating deep and inaccessible areas of the implant surface.
The complex surface micromorphology of the Laser-Lok implants was a fundamental implant system selection criteria for this study. The Laser-Lok surface located on the implant collar was found to have more residual ink accumulation after air abrasive therapies. In contrast, a previous in vitro study ${ }^{28}$ demonstrated that the machined surface collar was the easiest area to clean on an implant located within a circumferential defect, using different types of instruments (Gracey curette, ultrasonic scaler or air powder abrasive device). Therefore, despite a rough and complex surface on the coronal part of the implant may favor osseointegration and true, physical soft tissue attachment, ${ }^{29}$ the decontamination of this area may pose a challenging task toward the management of peri-implant diseases.

Although better decontamination was demonstrated using larger particles of greater density, such as SB $(40-65 \mu \mathrm{m})$, the use of smaller air abrasive particles such as glycine powder $(25 \mu \mathrm{m})$ and erythritol $(14 \mu \mathrm{m})$ remains an area of interest. Small particles are more likely to reach areas in the rough implant surface inaccessible by larger particles, and have a higher solubility potential which reduces the amount of undissolved particles in a water-air mixture that may be a contributing factor to enhanced surface abrasion ${ }^{23}$ and salt contamination. ${ }^{30}$ Moreover, we found that small particles only caused a minor effect on the implant topography as was consistent with previous studies. ${ }^{18}$ The reduced capacity of glycine and erythritol to remove implant contaminants found in our study can be detrimental for their use in the treatment of peri-implantitis; however, it has been demonstrated that the addition of antimicrobial agents to small abrasive particles provides not only adequate removal of bacterial biofilm, but also lessen the likelihood of future biofilm-associated infections. ${ }^{31}$ In another in vitro study, ${ }^{32}$ erythritol powder containing chlorhexidine was found to be as effective as SB in reducing Staphylococcus aureus and Aggregatibacter actinomycetemcomitans biofilm viability on preinfected titanium disks. The erythritol/chlorhexidine powder, however, showed higher post-treatment biofilm re-growth inhibition without titanium surface damage. Therefore, the use of small particles associated with antimicrobial agents may favor the prognosis of surgical procedures associated with peri-implantitis treatment due to reduced bacterial colonization.

An important finding in our study was the quantitative and qualitative demonstration that large-sized particles may change the implant surface topography. The SB powder was the only product that increased the surface roughness in the collar and threads significantly. Our SEM images reinforced these quantitative results in surface topography changes by showing the flattening of threads and the obliteration of the collar-to-thread transition after 60 seconds of large particles application. Limited number of in vitro studies provide quantitative assessment of surface roughness. ${ }^{19}$ Most of then failed to show quantitative differences in titanium roughness between $S B$ and smaller particles, ${ }^{32,33}$ and only two studies showed that the $R_{\mathrm{a}}$ value for SB was higher than glycine and untreated controls. ${ }^{19}$ Differently from any previous study, our implant decontamination model used a cutting-edge technology that evaluates changes in heights on the implant surface across various individual points in specific areas. This method allowed the assessment of the implant surface in two 
different areas, collar and threads, and the expression of results as area roughness $\left(S_{\mathrm{a}}\right)$ instead of linear roughness $\left(R_{\mathrm{a}}\right)$ as was shown in many previous studies assessing implant roughness. ${ }^{34,35}$ Early roughness meters could only measure $R_{\mathrm{a}}$, which is a dimensional unit expressed in micrometers that gives the surface roughness over a line (profile). This historical reason may explain why $R_{\mathrm{a}}$ became the most common roughness parameter in dental implant research. However, the development of new optical technologies such as TrueSurface Microscopy has allowed the measurement of surface roughness over an area. $S_{a}$ has already been considered as the preferred parameter for 3D measurements of implant surface. ${ }^{36}$ Using this method, all implants were scanned using white light interferometry to create 3D images that were used to calculate the implant roughness in different areas. Unlike $S_{a}, R_{a}$ values reduce the information of an entire surface in a profile analysis, therefore, care must be taken when interpreting them. The consistency of $R_{\mathrm{a}}$ is also questionable as very different values have been reported when similar surfaces were evaluated. ${ }^{36}$ Taking these limitations of profile roughness parameter into account, $S_{a}$ was considered a more accurate and reliable measurement of surface roughness to be used in this study and may become more common in implant topography analysis.

The increase in implant roughness may facilitate biofilm formation on dental implants exposed to the oral cavity. ${ }^{37}$ However, it is not possible to conclude that the amount of roughness change seen in this study can negatively affect re-osseointegration. Further studies are required to assess the impact of powders in the biocompatibility of the implant surface, and validate the relationship of implant roughness after air abrasive therapies and bone regeneration procedures.

\section{5 | CONCLUSION}

Within the limitations of the present in vitro model, larger sized powder particles such as SB had greater effectiveness in ink removal, but often caused greater damage to the implant surface. Glycine and erythritol were safe to use on implant surfaces as it resulted in only minor surface roughness changes; however, their cleaning capacity especially with erythritol was significantly lower than SB. The present study brought a novel method to assess the implant topography that provides accurate measurements of the surface roughness in different sites across the implant. Variations in the exposure time and the clinician skill were not included in this study as factors that may affect the efficiency of the decontamination process. Future in vivo studies are needed to validate the clinical efficacy of these air abrasive powders in removing biofilms, preventing bacterial colonization, and interfering with bone regeneration.

\section{ACKNOWLEDGMENTS}

The authors thank Dr Alexandra Suvorova from the UWA Centre for Microscopy, Characterisation \& Analysis (CMCA) for the technical support with the SEM, and Greg Sigglekow from UWA Dental School for helping with the $3 \mathrm{D}$ printed model fabrication. We also thank
BioHorizons for supplying the implant fixtures and EMS for providing the air abrasive powders used in the study.

\section{CONFLICT OF INTEREST}

The authors declare no conflict of interest.

\section{ORCID}

Victor H. Matsubara (D) https://orcid.org/0000-0003-3481-1621

Marcus J. L. Leong (D) https://orcid.org/0000-0001-7660-7536

Thomas Becker (D) https://orcid.org/0000-0002-4117-8249

Alessandro Quaranta (D) https://orcid.org/0000-0002-4225-9374

\section{REFERENCES}

1. Quirynen M, Herrera D, Teughels W, Sanz M. Implant therapy: 40 years of experience. Periodontol 2000. 2014;66:7-12.

2. Buser D, Mericske-Stern R, Bernard JP, et al. Long-term evaluation of non-submerged ITI implants. Part 1: 8-year life table analysis of a prospective multi-center study with 2359 implants. Clin Oral Implants Res. 1997;8:161-172.

3. Ellegaard B, Baelum V, Karring T. Implant therapy in periodontally compromised patients. Clin Oral Implants Res. 1997;8:180-188.

4. Lang NP, Wilson TG, Corbet EF. Biological complications with dental implants: their prevention, diagnosis and treatment. Clin Oral Implants Res. 2000;11(suppl 1):146-155.

5. Lindhe J, Berglundh T, Ericsson I, Liljenberg B, Marinello C. Experimental breakdown of peri-implant and periodontal tissues. A study in the beagle dog. Clin Oral Implants Res. 1992;3:9-16.

6. Araujo MG, Lindhe J. Peri-implant health. J Periodontol. 2018;89(suppl 1):S249-S256

7. Berglundh T, Lindhe J, Ericsson I, Marinello CP, Liljenberg B, Thomsen P. The soft tissue barrier at implants and teeth. Clin Ora Implants Res. 1991;2:81-90.

8. Schmidlin PR, Muller P, Attin T, Wieland M, Hofer D, Guggenheim B. Polyspecies biofilm formation on implant surfaces with different surface characteristics. J Appl Oral Sci. 2013;21:48-55.

9. Schwarz F, Herten M, Sager M, Bieling K, Sculean A, Becker J. Comparison of naturally occurring and ligature-induced peri-implantitis bone defects in humans and dogs. Clin Oral Implants Res. 2007;18: 161-170.

10. Figuero E, Graziani F, Sanz I, Herrera D, Sanz M. Management of peri-implant mucositis and peri-implantitis. Periodontol 2000. 2014; 66:255-273.

11. Grusovin MG, Coulthard P, Worthington HV, George P, Esposito M. Interventions for replacing missing teeth: maintaining and recovering soft tissue health around dental implants. Cochrane Database Syst Rev. 2010;CD003069.

12. Esposito M, Grusovin MG, Tzanetea E, Piattelli A, Worthington HV. Interventions for replacing missing teeth: treatment of periimplantitis. Cochrane Database Syst Rev. 2010;1:CD004970.

13. Tastepe CS, van Waas R, Liu Y, Wismeijer D. Air powder abrasive treatment as an implant surface cleaning method: a literature review. Int J Oral Maxillofac Implants. 2012;27:1461-1473.

14. Mizutani K, Aoki A, Coluzzi $D$, et al. Lasers in minimally invasive periodontal and peri-implant therapy. Periodontol 2000. 2016;71: 185-212.

15. Buchter A, Meyer U, Kruse-Losler B, Joos U, Kleinheinz J. Sustained release of doxycycline for the treatment of peri-implantitis: randomised controlled trial. Br J Oral Maxillofac Surg. 2004;42:439-444.

16. Fox SC, Moriarty JD, Kusy RP. The effects of scaling a titanium implant surface with metal and plastic instruments: an in vitro study. J Periodontol. 1990;61:485-490. 
17. Louropoulou A, Slot DE, Van der Weijden F. Influence of mechanical instruments on the biocompatibility of titanium dental implants surfaces: a systematic review. Clin Oral Implants Res. 2015;26:841-850.

18. Sahrmann P, Ronay V, Hofer D, Attin T, Jung RE, Schmidlin PR. In vitro cleaning potential of three different implant debridement methods. Clin Oral Implants Res. 2015;26:314-319.

19. Moharrami M, Perrotti V, laculli F, Love RM, Quaranta A. Effects of air abrasive decontamination on titanium surfaces: a systematic review of in vitro studies. Clin Implant Dent Relat Res. 2019;21:398-421.

20. Faggion CM Jr, Chambrone L, Listl S, Tu YK. Network meta-analysis for evaluating interventions in implant dentistry: the case of periimplantitis treatment. Clin Implant Dent Relat Res. 2013;15:576-588.

21. Romeo E, Ghisolfi M, Murgolo N, Chiapasco M, Lops D, Vogel G. Therapy of peri-implantitis with resective surgery. A 3-year clinical trial on rough screw-shaped oral implants. Part I: clinical outcome. Clin Oral Implants Res. 2005;16:9-18.

22. Hakki SS, Tatar G, Dundar N, Demiralp B. The effect of different cleaning methods on the surface and temperature of failed titanium implants: an in vitro study. Lasers Med Sci. 2017;32:563-571.

23. Wei MCT, Tran C, Meredith N, Walsh LJ. Effectiveness of implant surface debridement using particle beams at differing air pressures. Clin Exp Dent Res. 2017;3:148-153.

24. Quintero DG, Taylor RB, Miller MB, Merchant KR, Pasieta SA. Airabrasive disinfection of implant surfaces in a simulated model of periimplantitis. Implant Dent. 2017;26:423-428.

25. Heitz-Mayfield LJ, Mombelli A. The therapy of peri-implantitis: a systematic review. Int J Oral Maxillofac Implants. 2014;29(suppl): 325-345.

26. Taschieri S, Weinstein R, Del Fabbro M, Corbella S. Erythritolenriched air-polishing powder for the surgical treatment of periimplantitis. ScientificWorldJournal. 2015;2015:802310.

27. Schmidt KE, Auschill TM, Heumann C, et al. Influence of different instrumentation modalities on the surface characteristics and biofilm formation on dental implant neck, in vitro. Clin Oral Implants Res. 2017;28:483-490.

28. Steiger-Ronay V, Merlini A, Wiedemeier DB, Schmidlin PR, Attin T, Sahrmann $P$. Location of unaccessible implant surface areas during debridement in simulated peri-implantitis therapy. BMC Oral Health. 2017;17:137.

29. Guarnieri R, Rappelli G, Piemontese M, Procaccini M, Quaranta A. A double-blind randomized trial comparing implants with laser-microtextured and machined collar surfaces: microbiologic and clinical results. Int J Oral Maxillofac Implants. 2016;31:1117-1125.

30. Menini M, Piccardo P, Baldi D, Dellepiane E, Pera P. Morphological and chemical characteristics of different titanium surfaces treated by bicarbonate and glycine powder air abrasive systems. Implant Dent. 2015;24:47-56.

31. Drago L, Del Fabbro M, Bortolin M, Vassena C, De Vecchi $E$, Taschieri S. Biofilm removal and antimicrobial activity of two different air-polishing powders: an in vitro study. J Periodontol. 2014;85:e363e369.

32. Mensi M, Cochis A, Sordillo A, Uberti F, Rimondini L. Biofilm removal and bacterial re-colonization inhibition of a novel erythritol/ chlorhexidine air-polishing powder on titanium disks. Materials (Basel). 2018;11:1510.

33. Cochis A, Fini M, Carrassi A, Migliario M, Visai L, Rimondini L. Effect of air polishing with glycine powder on titanium abutment surfaces. Clin Oral Implants Res. 2013;24:904-909.

34. Cafiero C, Aglietta M, lorio-Siciliano V, Salvi GE, Blasi A, Matarasso S. Implant surface roughness alterations induced by different prophylactic procedures: an in vitro study. Clin Oral Implants Res. 2017;28: e16-e20.

35. Ramaglia L, di Lauro AE, Morgese F, Squillace A. Profilometric and standard error of the mean analysis of rough implant surfaces treated with different instrumentations. Implant Dent. 2006;15:77-82.

36. Wennerberg A, Albrektsson T. Suggested guidelines for the topographic evaluation of implant surfaces. Int J Oral Maxillofac Implants. 2000;15:331-344.

37. Subramani $\mathrm{K}$, Jung RE, Molenberg A, Hammerle $\mathrm{CH}$. Biofilm on dental implants: a review of the literature. Int J Oral Maxillofac Implants. 2009;24:616-626.

How to cite this article: Matsubara VH, Leong BW, Leong MJL, Lawrence Z, Becker T, Quaranta A. Cleaning potential of different air abrasive powders and their impact on implant surface roughness. Clin Implant Dent Relat Res. 2019; 1-9. https://doi.org/10.1111/cid.12875 